Abstract AB0841 - Table 1. Comparison of the perceived social support, depression and loneliness scores with the Morisky Green Levine Scale subgroups.

\begin{tabular}{|c|c|c|c|c|c|c|}
\hline \multirow[b]{2}{*}{ Characteristics } & \multicolumn{6}{|c|}{$\begin{array}{l}\text { Morisky Green Levine } \\
\text { medication adherence } \\
\text { groups }\end{array}$} \\
\hline & Overall & $\begin{array}{c}\text { High } \\
(n=43)^{a}\end{array}$ & $\begin{array}{l}\text { Medium } \\
(n=66)^{b}\end{array}$ & $\begin{array}{c}\text { Low } \\
(n=10)^{c}\end{array}$ & $\mathbf{F}$ & $\mathbf{p}$ \\
\hline $\begin{array}{l}\text { Multidimensional Scale of } \\
\text { Perceived Social Support total } \\
\text { score }^{12-84 *}\end{array}$ & $\begin{array}{c}63.52 \\
\pm 17.13\end{array}$ & $\begin{array}{r}65.09 \\
\pm 16.85\end{array}$ & $\begin{array}{r}64.65 \\
\pm 16.59\end{array}$ & $\begin{array}{r}49.30 \\
\pm 17.01\end{array}$ & 3.958 & 0.022 \\
\hline $\begin{array}{l}\text { Beck Depression Inventory (0- } \\
63)^{\star}\end{array}$ & $\begin{array}{r}13.65 \\
\pm 10.41\end{array}$ & $\begin{array}{r}13.07 \\
\pm 11.29\end{array}$ & $\begin{array}{l}12.15 \\
\pm 8.51\end{array}$ & $\begin{array}{r}26.10 \\
\pm 10.73\end{array}$ & 8.952 & $<0.001$ \\
\hline UCLA Loneliness Scale $\mathrm{e}^{20-80 *}$ & $\begin{array}{c}37.34 \\
\pm 11.22\end{array}$ & $\begin{array}{r}35.19 \\
\pm 11.26\end{array}$ & $\begin{array}{c}37.56 \\
\pm 10.90\end{array}$ & $\begin{array}{r}45.20 \\
\pm 10.56\end{array}$ & 3.388 & 0.037 \\
\hline
\end{tabular}

F: Oneway ANOVA, Statistically significant between a and $c$, and b and $c(p<0.05)$. ${ }^{*}$ It shows the lowest-highest scores that can be taken from the scale and its sub-dimensions.

Conclusions: In this study, it was shown that as the average age and social support scores of patients decreased, and as the BASDAI, spinal pain, spinal pain at night, fatigue, patient global assessment, loneliness and depression scores increased, adherence to treatment were decreased. It is thought that patients should be handled holistically in terms of biopsychosocial aspect in order to improve adherence to medical treatment.

Disclosure of Interest: None declared

DOI: 10.1136/annrheumdis-2018-eular.5873

\section{AB0842 DRUG SURVIVAL AND EFFECTIVENESS OF THE FIRST TNF INHIBITORS IN PATIENT WITH LATE ONSET SPONDYLARTHRITIS: TREASURE REAL-LIFE RESULTS}

N. Alpay Kanıtez ${ }^{1}$, S. Kiraz ${ }^{2}$, I. Ertenli² ${ }^{2}$ O. Küçükşahin ${ }^{2}$, E. Dalkılıç ${ }^{3}$, C. Bes ${ }^{1}$, T. Kaşifoğlü ${ }^{4}$, H. Emmungil ${ }^{5}$, S.S. Koca ${ }^{6}$, M. Çınar ${ }^{2}$, S. Yılmaz ${ }^{2}$, V. Yazısız ${ }^{7}$,

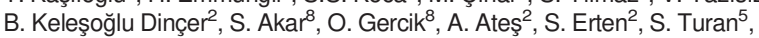
B. Yağız ${ }^{3}$, B. Öz ${ }^{6}$, S. Yaşar Bilge ${ }^{4}$, L. Kılıç ${ }^{2}$, G.K. Yardımcı ${ }^{2}$, B.N. Coşkun ${ }^{3}$ U. Kalyoncu ${ }^{2} .{ }^{1}$ TReasure biological registry, Istanbul; ${ }^{2}$ TReasure biological registry, Ankara; ${ }^{3}$ TReasure biological registry, Bursa; ${ }^{4}$ TReasure biological registry, Eskişehir, ${ }^{5}$ TReasure biological registry, Edirne; ${ }^{6}$ TReasure biological registry, Elazığ; ${ }^{7}$ TReasure biological registry, Antalya; ${ }^{8}$ TReasure biological registry, Izmir, Turkey

Background: Spondylartritis $(\mathrm{SpA})$ are usually observed in young patients and a clinical onset after 45 years is rare. The average life expectancy is getting longer and the proportion of late onset of SpA in rheumatology practice may became more common. SpA patients with late onset may have a distinctive clinical pattern in terms of functional impairment, extra-articular disease, co-morbidities and treatment status.

Objectives: The aim of this study was to compare the drug survival and effectiveness of tumour necrosis factor (TNF) inhibitors in patients with late onset SpA (LoSpA) compared with early onset SpA (EoSpA).

Methods: TReasure is a prospective, multicenter biological treatments registry from Turkey since 2016. It includes 15 different rheumatology centres. Patients with SpA fulfilling the ASAS criteria from TReasure database were divided into two groups as LoSpA (symptom onset $>45$ years of age) and EoSpA (symptom onset $\leq 45$ years of age). Drug retention rates of first TNF inhibitors were calculated using the time until drug discontinuation independent of the reason that drug interruption. Specific reasons for discontinuing drugs were also assessed.

Results: Of $1382 \mathrm{SpA}$ patients treated with TNF inhibitors, 9.4\% $(n=130)$ were included in the LoSpA and $90.6 \%(n=1252)$ were included in the EoSpA group. LoSpA had more female, enthesitis and psoriasis The median treatment duration was 53 months in LoSpA and 61 months in EoSpA. The baseline disease activity measures were similar except from ASDAS-ESH which is higher in LoSpA (table 1). The rate of major treatment response (BASDAI50) was lower in LoSpA than EoSPA at the last visit ( $26.1 \%$ vs $46.2 \%$; $p=0.009$ ). Regarding the survival rates of TNF inhibitors, there was no significant difference between the patient groups (figure 1). The major cause of discontinuation in both of groups was drug ineffectiveness $(68.6 \%$ in LoSpa and $60 \%$ in EoSpA, p>0.05).

Abstract AB0842 - Table 1

\begin{tabular}{lccc}
\hline & LoSpA n: 130 & EoSpA n:1252 & p values \\
\hline Age [median (min-max)] & $56,5(45-75)$ & $35(0-76)$ & $<0.001^{*}$ \\
Female n (\%) & $90(69)$ & $494(40)$ & $<0.001^{*}$ \\
Disease duration [median (min-max)] & $61(1-334)$ & $108(0-686)$ & $<0.001^{*}$ \\
BASDAI [median (min-max)] & $6.1(0-10)$ & $6(0-10)$ & 0.748 \\
BASFI [median (min-max)] & $5.3(0-10)$ & $4.5(0-10)$ & 0.098 \\
ASDAS-ESH [median (min-max)] & $3.29(1.23-4.92)$ & $2.91(0-5.7)$ & $0.001^{*}$ \\
ASDAS-CRP [median (min-max)] & $3.55(1.49-5.92)$ & $3.35(0.7-8)$ & 0.251 \\
\hline
\end{tabular}

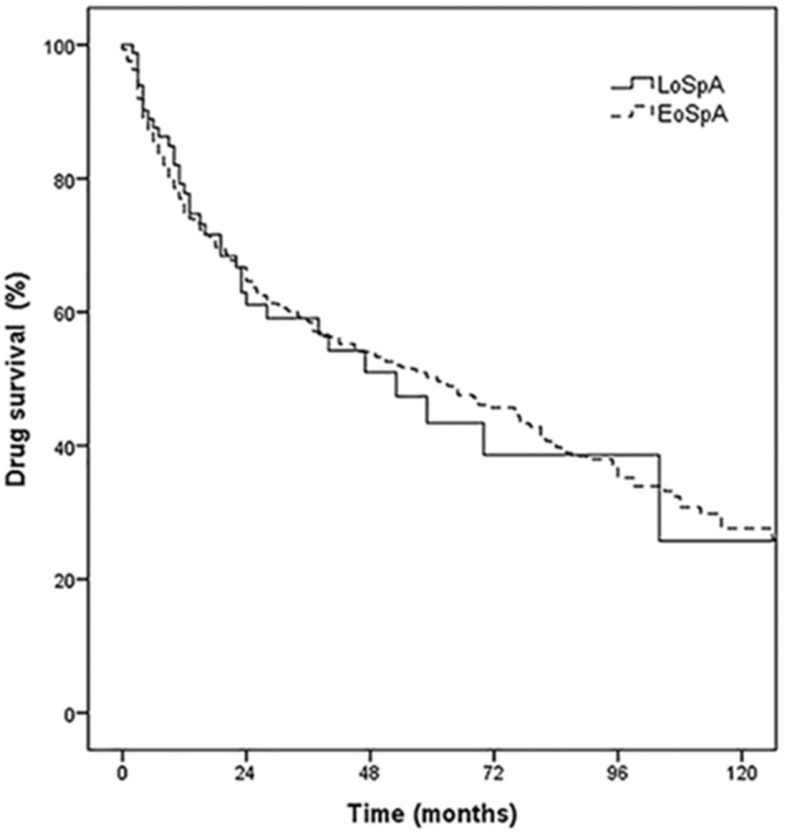

Abstract AB0842 - Figure 1

Conclusions: LoSpA patients were almost $10 \%$ of all biological registry. LoSpA patients were predominantly female, and they had relatively higher baseline disease activity and lower biological treatment response. On the other hand the drug survival rate and discontinuation reasons of TNF inhibitors in the LoSpA group was comparable to that in the younger group.

\section{REFERENCE:}

[1] - Chen HA, Chen CH, Liao HT, Lin YJ, Chen PC, Chen WS, et al. Clinical, functional, and radiographic differences among juvenile-onset, adult-onset, and late-onset ankylosing spondylitis. J Rheumatol. 2012; 39:1013-8

Disclosure of Interest: None declared

DOI: 10.1136/annrheumdis-2018-eular.4844

\section{AB0843 EFFECT OF TNF INHIBITORS ON BONE MICROARCHITECTURE IN PATIENTS WITH ANKYLOSING SPONDYLITIS: A LONGITUDINAL STUDY BASED ON HIGH-RESOLUTION PERIPHERAL QUANTITATIVE BASED (HRPQCT)}

N. Nigil Haroon ${ }^{1}$, E. Szabo ${ }^{2}$, A.M. CHEUNG ${ }^{3}$, R. Inman ${ }^{4} .{ }^{1}$ Medicine, Northern Ontario School of Medicine, Sudbury; ${ }^{2}$ Osteoporosis program, UHN; ${ }^{3}$ Medicine, Univ of Toronto; ${ }^{4}$ Medicine, unit of Toronto, Toronto, Canada

Background: Ankylosing spondylitis (AS) is associated with high risk of fractures. BMD, bone microarchitecture and strength are negatively affected in AS. TNF inhibitors such as etanercept, adalimumab, golimumab and infliximab are the mainstay of treatment in AS. However no data is available on the effect of TNF inhibitors on bone microarchitecture and strength.

Objectives: This study aimed to assess the effect of TNF inhibitors on bone microarchitecture in patients with AS.

Methods: AS was defined by Modified New York criteria. Areal BMD was meas ured by DXA. Volumetric BMD (vBMD) and bone microarchitecture were measured using highresolution peripheral quantitative $C T$ (HRPQCT) at the radius and tibia at baseline and after one year of treatment with TNF inhibitors. Intake of calcium and vitamin D were optimised.

Results: There were 31 subjects ( $58 \%$ men). Mean (+SD) age and BASDAI were $40+14$ years and $4.1+2.1$ respectively. Median duration of disease was $14(\mathrm{IQ}$ : 6.5-25.5) years. Mean duration of follow-up was 15 months. Areal BMD $(n=22)$ at lumbar spine $(1.053+0.235$ vs. $1.049+0.202, p=0.89)$, total hip $(0.944+0.152$ vs $0.912+0.164, p=0.5)$, and femoral neck $(0.955+0.151$ vs. $0.954+0.191, p=0.2)$ did not change significantly. HRpQCT $(n=31)$ on follow-up demonstrated that total, trabecular and cortical volumetric BMD were unchanged at both radius and tibia (table 1). Also, HRpQCT based trabecular parameters such as trabecular number, thickness and separation, BV/TV and cortical parameters such as cortical porosity and thickness remained stable (table 1). FEA estimates of bone stiffness and 